\title{
Prescription Pattern of Antibiotics in Paediatric Patients in a Rural Teaching Hospital of North India
}

\author{
Ankush Malik', Parminder Nain ${ }^{1}$, Baljeet Maini' ${ }^{2}$, Shishpal ${ }^{1}$, and Jaspreet Kaur1 \\ ${ }^{1}$ Department of Pharmacy Practice, M.M. College of Pharmacy, Maharishi Markandeshwar (Deemed to be \\ University), Mullana-Ambala, Haryana, India \\ ${ }^{2}$ Department of Paediatrics, M.M.I.M.S.R, Maharishi Markandeshwar (Deemed to be University), Mullana- \\ Ambala, Haryana, India
}

\author{
Correspondence: \\ Jaspreet Kaur \\ Department of Pharmacy Practice, \\ M.M. College of Pharmacy, \\ Maharishi Markandeshwar (Deemed to \\ be University), \\ Mullana-Ambala, Haryana, India \\ Email: preetisidana@gmail.com
}

DOI: $10.3126 /$ jnps.v41i2.31498

Submitted on: 2020-07-18

Accepted on: 2021-02-20

Acknowledgements: We would like to acknowledge of Maharishi Markandeshwar (Deemed to be University) and medical professionals / staff of Paediatric department of MMIMSR for assistance with patient evaluation

Funding: Nil

Conflict of Interest: None declared

Permission from IRB: Yes

To cite this article: Malik A, Nain $P$, Shishpal, Maini B, Kaur J. Prescription Pattern of Antibiotics in Paediatric Patients in a Rural Teaching Hospital of North India. J Nepal Paediatr Soc. 2021;41(2):211-7.

\section{ABSTRACT}

Introduction: Due to complex dosage calculations, requirement and different pharmacokinetics profile of drugs in paediatric population, there is a risk of development of drug resistance due to the excessive and inappropriate use of antibiotics. The aim of this study is to determine the antibiotic prescriptions pattern and analyse the rationale use of antibiotic in paediatric patient.

Methods: A prospective observational study was carried out for the period of six months. Data was obtained from the records of paediatric patients between six months to 12 years of age who visited the hospital for febrile illnesses. Appropriateness of the prescriptions was evaluated by considering various parameters like selection of antibiotics (adherence to standard treatment protocols of childhood febrile illnesses), dose, dosage form, route, frequency and duration of antibiotic administration. Quantitative data was analysed in terms of percentage and graphs.

Results: One hundred and fifty prescriptions in a successive manner from paediatric department were collected and analysed, out of which $40 \%$ were male \& $60 \%$ were female cases. The mean age of the patients was $5.9 \pm 3.3$ years. The range of duration of antimicrobial therapy was $3-9$ days $(6.03 \pm 3.53$ days). Sixty six percent of paediatric patients were on single antibiotic and most of the paediatric patients were receiving parenteral preparation. The antibiotic treatment was given on the basis of definitive diagnosis in $52 \%$ and empirical therapy in $46 \%$ cases. Antibiotic prescribed were from class cephalosporin $(80 \%)$ followed by penicillin $(18 \%)$ and microcline $(14 \%)$, glycopeptides $(2 \%)$ and nitroimidazoles $(2 \%)$.

Conclusions: Febrile children received antibiotics on an empirical basis, in accordance with standard treatment protocols. Cephalosporins are the major class of drugs prescribed.

Key words: Antibiotic; Paediatric; Prescription Pattern

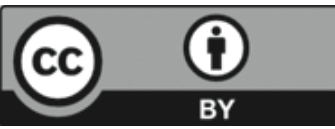

This work is licensed under creative common attribution 3.0 license 


\section{INTRODUCTION}

Among various categories of drugs, antibiotics are the most frequently prescribed medications in modern medicine in developing countries. Today, over 100 different antibiotics are available and play an important role in management of wide variety of life-threatening infections. ${ }^{1}$ Antibiotics are vital in today's medical practice. However due to the overuse and inappropriate use of antibiotics there is an increase development of bacterial resistance, cost of treatment and unnecessary side effects. ${ }^{2}$ It is important to have a rational approach to antibiotic use. Most of the infections in children are caused by viruses and do not need antibiotics. ${ }^{3}$

Antibiotic usage depends on ability of health professionals to differentiate and discriminate among the various choices of drugs and their adherence to standard treatment protocols. ${ }^{4}$ Study of antibiotic prescription practices is a part of enhancement of the medical standards in a health organisation and society at large. ${ }^{5}$ The observance of standards of medication is done from time to time in all large hospitals. The studies of prescribing pattern, a part of medical audit, seeks to evaluate, monitor and if possible, suggest modification in prescribing practices to make medical care rational and cost effective. ${ }^{6-7}$

The pharmacokinetics profile of the paediatrics is different from adults. This alters the dosage requirements in this population otherwise they lead to fatal effects. ${ }^{8-9}$ Paediatric physicians at times too include antibiotics in an irrational manner. Therefore, an effective step should be taken for rational and effective use of antibiotics especially in paediatric population. ${ }^{10}$

Prescribing drugs is an important skill which needs to be continuously assessed and refined accordingly. So there is always a chance of irrational drugs in the prescription due to various reasons. The assessment of the prescription will help to know the attitude of the physicians towards prescribing and to provide rationality in the prescription. Rationality of the prescription helps the physician to upgrade the knowledge and improves attitude towards selecting the most appropriate cost-effective treatment. ${ }^{11}$ Knowledge of prescribing patterns is useful to plan relevant activities that limit non rational use of antibiotics. ${ }^{12}$
Present study was aimed to analysis of antibiotic prescription pattern to treat the febrile and non febrile infections in paediatric department of our tertiary care hospital.

\section{METHODS}

This was a prospective observational study conducted by collecting the prescriptions of paediatric patients of age between six months to 12 years. The participants enrolled in the study only after filling a properly written informed consent. The institutional ethical committee permission was taken prior to conduct this study. Basic demographic information of patients and clinical details were noted in a structured performa. Details of prescribed antibiotics on the basis of pharmacological details like dose, route, frequency of administration, diseases for which they were indicated, were documented in all patients. Selection of antibiotics was also assessed for appropriateness with respect to microbiological and other laboratory panel reports. Assessment of drug interactions and number of antibiotics prescribed in each prescription were also recorded. The choice of antibiotics was also studied with respect to national antibiotic use policy laid down by Government of India. ${ }^{13}$ All the data were exported to Microsoft Excel spread sheets. The results were presented as percentage and mean \pm Standard deviation (SD).

\section{RESULTS}

The study was carried out to assess the antibiotic use and prescribing pattern by medical practitioner in infectious disease. In this study analysis, a total of 150 infectious disease patients were selected on the basis of W.H.O. guidelines. Hence the result was based on the data of 150 patients.

Demographic Data of Paediatric Patients: The sample of study $(\mathrm{N}=150)$ had predominantly female population $(60 \%)$ as compared to male population $(40 \%)$.

Table 1 suggests that more number of patients were in between 1 - 4 years of age (38\%) followed by number of patients between $9-12$ years of age $(28 \%)$. Least number of patients were in age group between 5 - 8 years of age (24\%) followed by age $6-12$ months of age (10\%). More number of 
Table 1. Demographic data of Paediatric Patients

\begin{tabular}{|c|c|}
\hline & Number of Patients \\
\hline \multicolumn{2}{|l|}{ Gender (Sex) } \\
\hline Male & $60(40 \%)$ \\
\hline Female & $90(60 \%)$ \\
\hline Total Patients & 150 \\
\hline \multicolumn{2}{|l|}{ Age } \\
\hline $\begin{array}{l}\text { Six months to } 12 \\
\text { months }\end{array}$ & $15(10 \%)$ \\
\hline 1 Year to 4 Year & $57(38 \%)$ \\
\hline 5 Year to 8 Year & $36(24 \%)$ \\
\hline 9 Year to 12 Year & $42(28 \%)$ \\
\hline Age $($ mean \pm SD $)$ & $5.9 \pm 3.3$ \\
\hline \multicolumn{2}{|l|}{ Area } \\
\hline Rural & $97(64 \%)$ \\
\hline Urban & $53(36 \%$ \\
\hline
\end{tabular}

patient were from rural (64\%) as compared to urban area (36\%).

In present study, bronchitis (22\%), followed by meningitis (14\%), Acute Gastroenteritis (10\%), UTI (18\%), septicaemia (06\%) were the chief infectious etiology (Figure 1).

The results suggests that maximum number of patients prescribed mono-therapy (66\%) followed dual therapy (32\%). The least number of patients on triple therapy (2\%) of antibiotic (Figure 2). It also found that treatment was given on the basis of definitive therapy (52\%) and empirical therapy (48\%) (Table 2).

Table 2. Pattern of Antibiotic Prescribed

\begin{tabular}{|lrr|}
\hline $\begin{array}{l}\text { Antibiotic } \\
\text { Pattern }\end{array}$ & $\begin{array}{c}\text { Number of } \\
\text { Prescription }\end{array}$ & $\begin{array}{c}\text { Prescription } \\
\text { (\%) }\end{array}$ \\
\hline Mono therapy & 100 & $66 \%$ \\
Dual therapy & 47 & $32 \%$ \\
Triple therapy & 3 & $2 \%$ \\
$\begin{array}{l}\text { Empirical } \\
\text { therapy }\end{array}$ & 72 & $48 \%$ \\
$\begin{array}{l}\text { Definitive } \\
\text { therapy }\end{array}$ & 78 & $52 \%$ \\
\hline
\end{tabular}

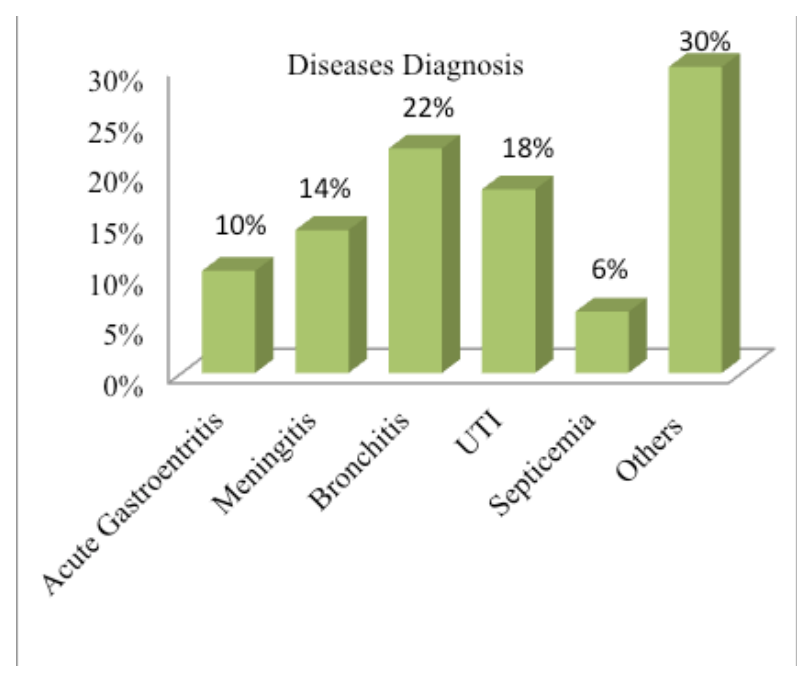

Figure 1. Pattern of infection Diagnosis

In this study maximum antibiotic prescribed by physician was from class cephalosporin (80\%) followed by penicillin (18\%) and macrolide (14\%), but least number of antibiotic prescribed from class of glycopeptides $(2 \%)$ and nitroimidazole $(2 \%)$.

During this study, the antibiotics predominately prescribed under different categories (Figure 3). The maximum prescribed antibiotics was ceftriaxone $(87.5 \%)$ in case of meningitis patients, ceftriaxone $(60 \%)$ in case of UTI patients, amikacin $(50 \%)$, ceftriaxone $(50 \%)$ in case of septicemic patients, ceftriaxone (43\%) in case of Gastroenteritis patients, ceftriaxone $(35 \%)$ in case of bronchitis, ampicillin (30\%) in case of UTI patients, vancomycin $(28.5 \%)$ in case of Gastroenteritis, azithromycin (21\%) and amoxicillin (21\%) in case of bronchitis,

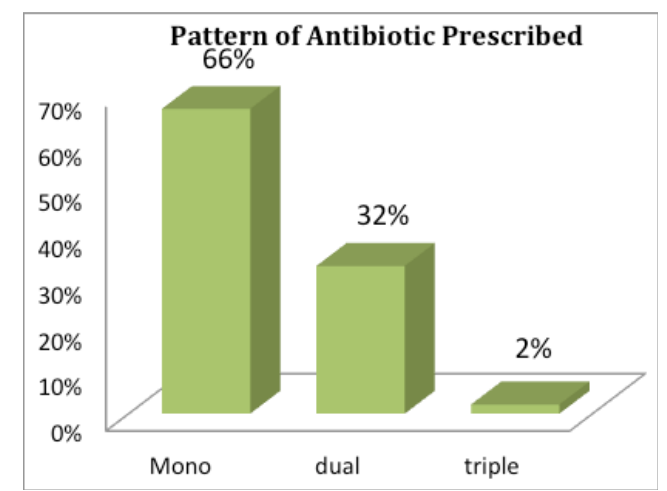

Figure 2. Pattern of of Antibiotic Prescribed 
Table 3. Distribution of Antibiotic Class Prescribed by Physician

\begin{tabular}{|lrr|}
\hline Class of Antibiotics & N=150 & Ab (\%) \\
\hline Cephalosporin & 119 & 80 \\
Amino glycoside & 12 & 8 \\
Macrolide antibiotics & 21 & 14 \\
Penicillin & 27 & 18 \\
Glycopeptides antibiotics & 3 & 2 \\
Nitroimidazole & 3 & 2 \\
\hline
\end{tabular}

ciprofloxacin (14.5\%) and ampicillin (14.5\%) in case of gastroenteritis, azithromycin $(12.5 \%)$ in case of meningitis patients, cefixime $(10 \%)$ in case of UTI patients, amoxicillin with clavulanic acid (7.1\%), amikacin (7.5\%) and cloxacillin with ampicillin (7.5\%).

The table 4 showed that the maximum duration/ length of antibiotic prescribed days were 1 - 5 days and duration of 10 days or more was recorded least.

\section{DISCUSSION}

The inappropriate and excessive use of antibiotics is a major public health issue. This study focused
Table 4. Duration of Antibiotic Prescribed (In Days)

\begin{tabular}{|lr|}
\hline Duration & \%Antibiotics \\
\hline 1 - 5 Days & $53 \%$ \\
6 - 10 Days & $28 \%$ \\
$>10$ Days & $19 \%$ \\
\hline
\end{tabular}

on antibiotic prescriptions for paediatric patients in terms of average time of antibiotic therapy, the range of antibiotics used and the appropriateness of the antibiotic utilisation.

There are problems of raised health-care costs and the development of antibiotic resistance associated with irrational antibiotic use. ${ }^{2}$ Due to these emergent threats it is essential to study the patterns of antibiotic use from time to time.

In present study, the demographic sample of study $(\mathrm{N}=150)$ indicates that female population were found to be predominantly having more infection $(60 \%)$ as compared to male population $(40 \%)$. Majority of children were in the age group of 1 - 4 years (38\%), followed by children of age between 9 - 12 years (28\%). Least number of children were in age group 6 - 12 months (10\%). Another study

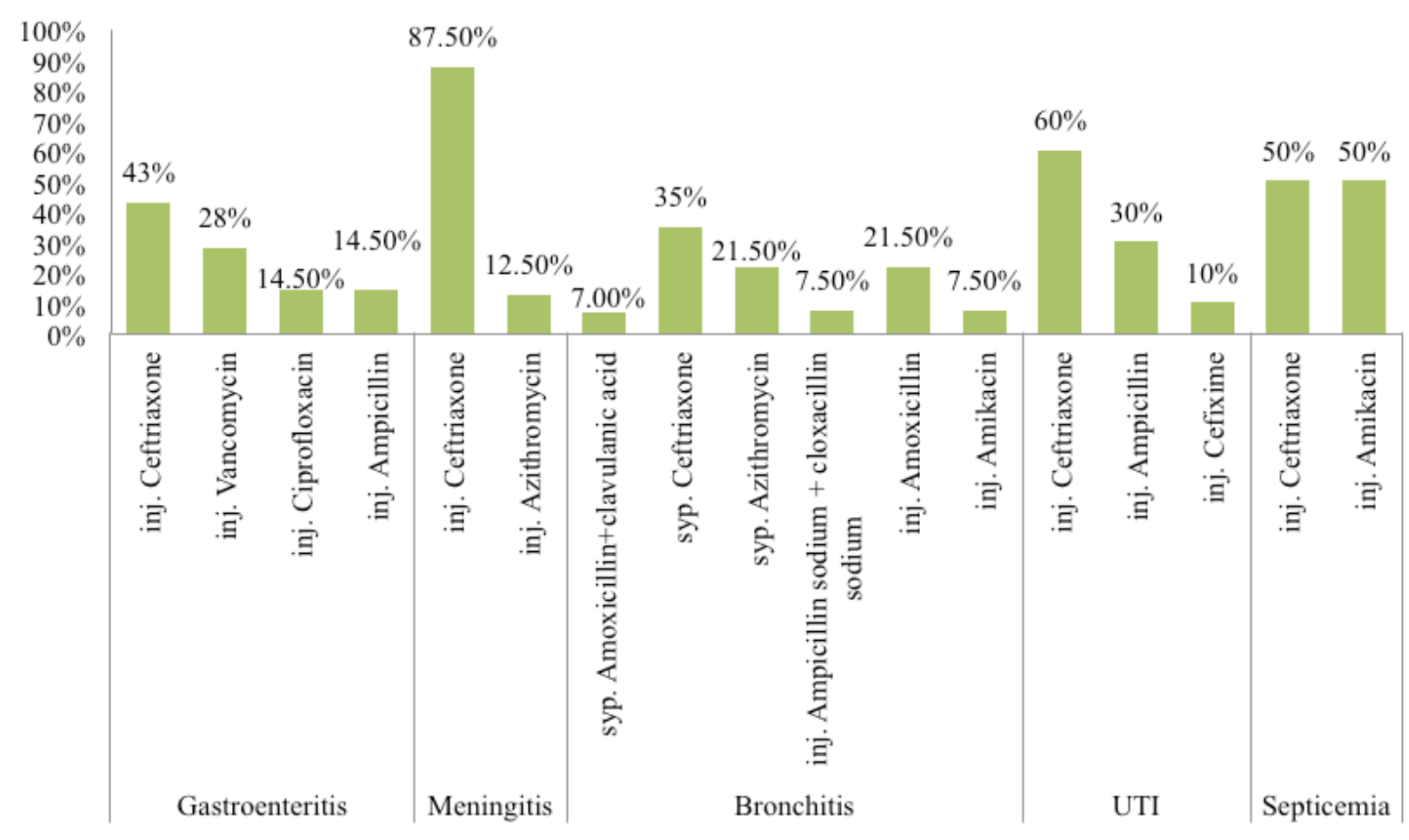

Figure 3. Choice of antibiotics prescribed on the basis of infectious disease 
conducted by Van Houten et al.,1998, showed children less than two years received antibiotics more frequently on a clinical basis, without conformation of a bacterial infection. ${ }^{14}$ Result of this study show use of antibiotics more frequently in small age group than the older children.

In over $46 \%$ of 150 antibiotic prescriptions were for children diagnosed with bronchitis (respiratory tract infection), $(22 \%)$, followed by urinary tract infection (UTI) (18\%), meningitis (14\%) acute gastroenteritis $(10 \%)$ and least with septicemia (6\%). Results revealed that ceftriaxone, ciprofloxacin, ampicillin were prescribed for gastroenteritis. The result of another similar study, reported similar use in case of invasive gastroenteritis. ${ }^{15}$ In previous studies, URTIs and bronchitis were identified as the diagnosis most frequently associated with inappropriate antibiotic use. ${ }^{16}$ In this study similar bronchitis is much more common which shows a maximum use of inappropriate antibiotics for these conditions.

Majority of the children were prescribed with single antibiotic $(66 \%)$, followed by dual therapy in 47 number of patients (32\%) of antibiotic prescribed. In only three patients triple therapy was prescribed $(2 \%)$. Patients with more than one antibiotics use were those with serious underlying medical conditions like meningitis and sepsis. Antibiotics were prescribed for duration of 1 - 5 days in children (53\%) followed by 6 - 10 days $(28 \%)$ and more than 10 days in children (19\%). In present study, the mean value of antibiotic duration/ length in children with prescribed antibiotic therapy was seven days. The longest duration of antibiotic therapy was 14 days and the shortest durations of therapy was three days. Similar to the results of a previously done study, length of antibiotics for the management of infection ( $>10$ days) was in complicated serious cases and the shortest length of antibiotic exposure was three day in minor illnesses. ${ }^{17}$

The culture test was done in total 98 children. Out of these, blood culture was done in 60 children and urine culture in rest of 38 children. Positive culture test were obtained 48 in blood cultures and 30 in urine cultures. Indication for starting antibiotics was definitive in $52 \%$, followed by empirical in $48 \%$ children. Most of the prescription in this study contained the antibiotics given on empirical basis $(48 \%)$ i.e. without the confirmation of the diagnosis in accordance with national standard antibiotic policy. This finding is similar to the study conducted diagnosis in over $50 \%$ of the IPD/OPD children. ${ }^{18}$

This study also identified a substantial use of $3^{\text {rd }}$ generation cephalosporin in gastroenteritis, UTI and meningitis which is similar to various studies, which reported the use of broad spectrum $3^{\text {rd }}$ generation of antibiotic in approximately one third of the UTI in standard antibiotic treatment visits. ${ }^{19-21}$ In this study, we identified nine nonsignificant drug interactions i.e. two minor and six moderate drug interactions.

Limitations of this study is that it was carried out in months of winter season, thereby the infectious disease profile and antibiotic use may be limited in description. We recommend that such studies / surveys should be carried out regularly and extended to private clinics and nursing homes who cater to health needs of a majority of Indian population.

\section{CONCLUSIONS}

A high percentage of infected children received antibiotics on an empirical basis, pending the results of bacterial culture, as per standard treatment protocols. There was complete adherence to the prescribed medication; no significant drug interaction and no error of administration were found in the study. This study adds to the knowledge of antibiotic usage by paediatricians. Pharmacist plays a major role in monitoring, adherence of drug according to the guidelines, and drug interaction. Involvement of pharmacist in paediatric prescription can limit the unnecessary usage of antibiotics and thus problems associated with them. We recommend larger studies in general population community setting. 


\section{REFERENCES}

1. Fair RJ, and Tor Y. Antibiotics and bacterial resistance in the 21st century. Perspect Medicin Chem. 2014;6:25-64. DOI:10.4137/PMC.S14459

2. Llor C, and Bjerrum L. Antimicrobial resistance: risk associated with antibiotic overuse and initiatives to reduce the problem. Ther Adv Drug Saf. 2014;5(6):229-241. DOI:10.1177/2042098614554919.

3. Leekha S, Terrell CL, Edson RS. General principles of antimicrobial therapy. Mayo Clin Proc. 2011;86(2): 156-67. DOI:10.4065/mcp.2010.0639.

4. Crockett AB. Use of prescription drugs: rising or declining? Nurs Clin North Am. 2005;40(1):33-49. DOI: 10.1016/ j.cnur.2004.08.012. PMID: 15733945 .

5. Patterson HR, J R Coll. Gen Practice- Problems of audit and research. J R Coll Gen Pract. 1986;36:286-196. PMID: 3746760

6. Badar VA, Navale SKB. Study of prescribing pattern of antimicrobial agents in medicine intensive care unit of a teaching hospital in Central India. J Assoc Physicians Ind. 2012;60:20-3. PMID: 23029737

7. Amadeo B, Zarb P, Muller A, Drapier N, Vankerckhoven V, Rogues AM, Davey P, Goossens H. ESAC III Hospital Care Subproject Group. European Surveillance of Antibiotic Consumption (ESAC) point prevalence survey 2008: paediatric antimicrobial prescribing in 32 hospitals of 21 European countries. J Antimicrob Chemother. 2010; 65(10): 2247-52. DOI: $10.1093 / \mathrm{jac} / \mathrm{dkq} 309$.

8. Parthasarathi G, Karin Nyfort-Hansen, Milap C Nahata. A Text book of Clinical Pharmacy Practice. First Edition 2004, Orient Longman private Ltd.

9. Ferro A. Paediatric prescribing: why children are not small adults. Brit J Clin Pharmac. 2015;79:3:351-3. DOI: 10.1111/bcp. 12540.

10. Ramanath KV, Balaji B. Study the outpatients' prescription pattern of antibiotics in paediatric populations of two hospitals. Arch Pharma Pract. 2013;4:21-7. DOI: 10.4103/2045-080X.111578

11. Batta A, Singh B. Rational approach to prescription writing: A preview. Neurol India. 2018;66:928-33. DOI: 10.4103/0028-3886.236960.

12. De With K, Allerberger F, Amann S. Strategies to enhance rational use of antibiotics in hospital: a guideline by the German Society for Infectious Diseases. Infection. 2016; 44(3):395-439. DOI:10.1007/s15010-016-0885-Z

13. Aggarwal S, Walia K, Madhumathi J, Gopalkrishnan R, Ohri V, Gangakhedkar R. Treatment Guidelines for Antimicrobial Use in Common Syndromes 2019. 2nd edition, ICMR, New Delhi. Available from: http:// www.ijmm.org/documents/Treatment_Guidelines_2019_Final.pdf

14. Van Houten MA, Luinge K, Laseur M, Kimpen JL. Antibiotic utilisation for hospitalised paediatric patients. Int J Antimicrob Agents. 1998;10:161-4. DOI: 10.1016/s0924-8579(98)00022-3.

15. Palikhe N. Prescribing pattern of antibiotics in paediatric hospital of Kathmandu valley. Kathmandu Univ Med J. 2004;2(1):6-12. PMID: 19780281.

16. Wigton RS, Darr CA, Corbett KK, Nickol DR, Gonzales R. How do community practitioners decide whether to prescribe antibiotics for acute respiratory tract infections? J Gen Intern Med. 2008;23(10):1615-20. DOI:10.1007/ s11606-008-0707-9

17. Li B, Webster TJ. Bacteria antibiotic resistance: New challenges and opportunities for implant-associated orthopedic infections. J Orthop Res. 2018;36(1):22-32. DOI:10.1002/jor.23656.

18. Mettler J, Simcock M, Sendi P. Empirical use of antibiotics and adjustment of empirical antibiotic therapies in a university hospital: a prospective observational study. BMC Infect Dis. 2007;7(2)1-5. DOI: https://doi.org/ 10.1186/1471-2334-7-21

19. Shimels T, Bilal AI, Mulugeta A. Evaluation of Ceftriaxone utilisation in internal medicine wards of general hospitals in Addis Ababa, Ethiopia: a comparative retrospective study. J Pharm Policy Pract. 2015;8:26. DOI: 10.1186/s40545-015-0047-1

20. Riaz B, Khatoon H. Evaluation of the use of cephalosporin antibiotics in paediatrics. J Appl Pharmac Sci. 2013;3 (04):63-6. DOI: 10.7324/JAPS.2013.3411. 
21. Woo B, Jung Y, Kim HS. Antibiotic Sensitivity Patterns in Children with Urinary Tract Infection: Retrospective Study Over 8 Years in a Single Center. Child Kidney Dis. 2019;23(1):22-8. DOI: https://doi.org/10.3339/jkspn. 2019.23.1.22 